\title{
The basic anatomy of the optic nerve and visual system (or, why Thoreau was wrong)
}

\author{
Eric A. Kelts* \\ Porter Adventist Hospital, Denver, CO, USA
}

\section{Introduction}

Humans are visual creatures. With an estimated $35 \%$ of our brain devoted to vision, and 1.1 million axons associated with each of our optic nerves, it is hard to overestimate how important the visual system is to our ability to be Homo sapiens. This has become increasingly true in recent centuries, as entertainment has evolved from being primarily auditory in nature to primarily visual reflected in the transition from spoken tales to radio, and then to television and movies.

The ability of the eye to see is dependent on the health of the optical system which focuses light on the retina; the health of the retina itself, which translates light into recognizable objects and allows us to visually interact with our world; and the health of the occipital cortex of the brain, which interprets the raw data provided by the optical system of the eye as transmitted through the conduit that is the optic nerves, tracts, and radiations.

The eye itself is a complex optical structure, made up of a sophisticated focusing system, a structural matrix to support its shape, and a photoreceptor layer and its supporting cell layer. In fact, it is the sheer complexity of this system that has led to many of the controversies in the debate between to proponents of the theorem of Evolution/Natural Selection and the supporters of the

*Address for correspondence: Eric Kelts, 7400 East Arapahoe Road Suite \#100, Centennial, CO 80112, USA. E-mail: ekeltsmd@ yahoo.com.

hypothesis of Intelligent Design, but that is a story for another time and place [1].

The focusing system has three primary parts, the cornea (which provides the majority of the eye's refractive power as the interface between air, the fluid layers of the tear film and the tissue layers of the corneal stratum), the iris, and the lens and ciliary muscle. As a result, light entering the eye is initially focused by the cornea through the small pupillary aperture protected by the iris, then refocused on the back of the eye by the lens and the ciliary muscle. This complex system allows for alterations in focus, allowing the eye to alter its focus between near and infinity (in an emmetropic eye).

To keep the structures of the eye intact, there is a complex layer of scaffolding for the eyeball itself. Covering the eye and protecting its outer surface are the conjunctiva and lid apparatus. Below the conjunctiva lies the sclera (which is anatomically contiguous with the cornea), the uveal tract (including the choroid), and the nervous layer (including the retina). However, internally the structure of the eye is supported by the vitreous (a thick gel made up of $98 \%$ water and hyaluronic acid) in the posterior chamber, and the aqueous humor (similar to plasma) in the anterior chamber.

The retina, optic nerve and retinal pigment epithelium make up the photoreceptor layer and its supporting cells. The retina is made up of 8 distinct functional layers and two membranes (Fig. 1). Travelling from the vitreous interface posteriorly, the layers are: 1) nerve fiber layer, 2) ganglion cell layer, 3) inner plexiform layer, 4) inner nuclear layer, 5) outer plexiform layer, 6 ) outer nuclear layer, 7) photoreceptor layer (rods and cones), and 8) retinal pigment epithelium [2]. Note that this description does not include the internal limiting membrane (ILM) and external limiting membrane 


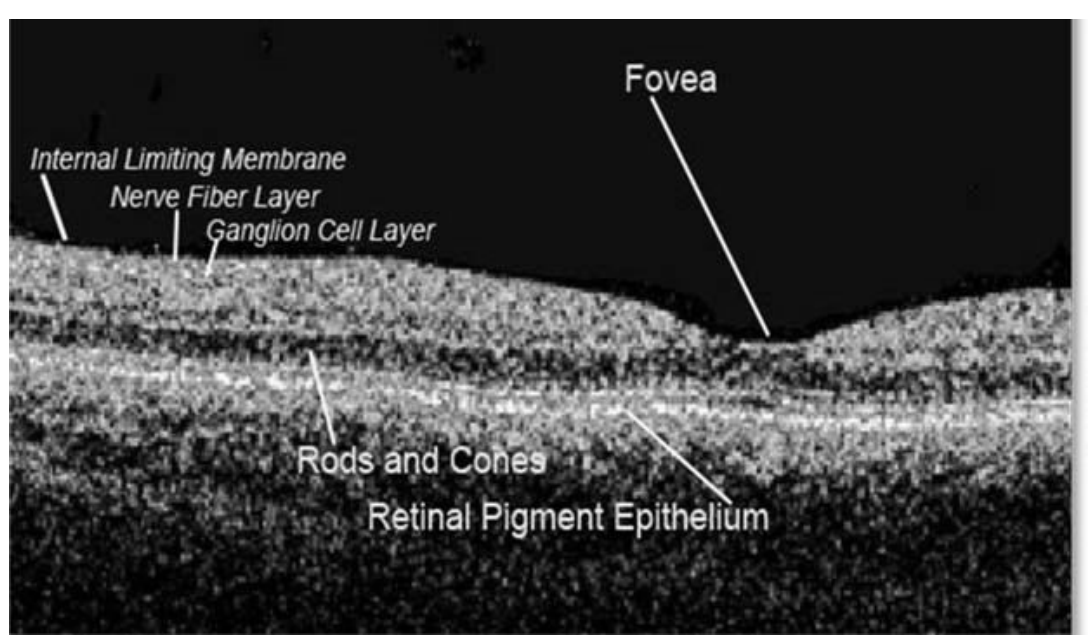

Fig. 1. OCT Image of the fovea showing the internal limiting membrane, nerve fiber layer, ganglion cell layer, rods/cones and retinal pigment epithelium.

(ELM), which are also parts of the retina. Each layer serves a distinct function.

The complex system that is the retina itself is unable to meet all of its metabolic needs from the arterioles and capillaries of the retinal artery, however. The deeper layers of the retina, including the photoreceptors, require the direct support of another dedicated layer of the eye. The retinal pigment epithelium (RPE) provides this nutritional support for the deeper retinal layers, and also acts to remove waste products from the environment of the photoreceptor cells.

However, from the standpoint of our discussion, the most important two layers of the retina are the ganglion cell layer and the nerve fiber layer, as these serve as the foundation for the optic nerve itself.

The ganglion cell layer contains the cell bodies for the axons of the optic nerve. The nerve fiber layer contains these axons as they stream across the surface of the retina just beneath the footplates of the Muller cells which coalesce to form the structure of the internal limiting membrane. The fibers join together at the optic disc, where they are bundled together, pass through the 200-300 fenestrations of the lamina cribrosa, and are redirected posteriorly out of the eye (Fig. 2). Interestingly, the size of the scleral canal through which the axons pass is directly responsible for the size of the optic disc and cup [3].

Once the axons of the optic nerve leave the eye, they form the orbital portion of the optic nerve. This segment, which is approximately $25 \mathrm{~mm}$ in length, is longer than needed to pass from globe to orbital apex [4]. This allows the eye to move freely within the orbit without restriction. The nerve is surrounded by the thick, fibrous sheath known as the dura, which is contiguous with the dura surrounding the brain, allowing CSF to flow freely up to the back of the eye and back into the intracranial vault. It is this connection that allows increased cerebrospinal fluid pressure to be transmitted to the back of the eye to produce papilledema.

The axons next pass through the optic foramen at the apex of the orbit (Fig. 3). By contrast, the ophthalmic artery and sympathetic fibers pass into the orbit simultaneously with the exiting axons of the optic nerve.

The optic canal is a bone-encased tunnel which is intended to protect the optic nerve. The axons of the optic nerve enter the optic canal, which runs for approximately $10 \mathrm{~mm}$ before opening into the middle cranial fossa to form the intracranial portion of the optic nerve. This segment again runs for about $10 \mathrm{~mm}$ before the two optic nerves join together to form the optic chiasm adjacent to and directly behind the stalk of the pituitary gland [5].

The optic chiasm is a complex intersection of both optic nerves. Slightly more than half of the axons in each optic nerve cross to the other side, splitting the axons corresponding to homologous portions of the visual fields of each eye and placing them together before sending them onward to form the optic tracts. As such, the left half of each visual field passes into the right optic tract, and the right half of both visual fields becomes the left optic tract. This unexpected anatomic anomaly helps eye care professionals and neurologists in their assessment of visual system trauma, by producing the well-described homonymous visual field defects result- 


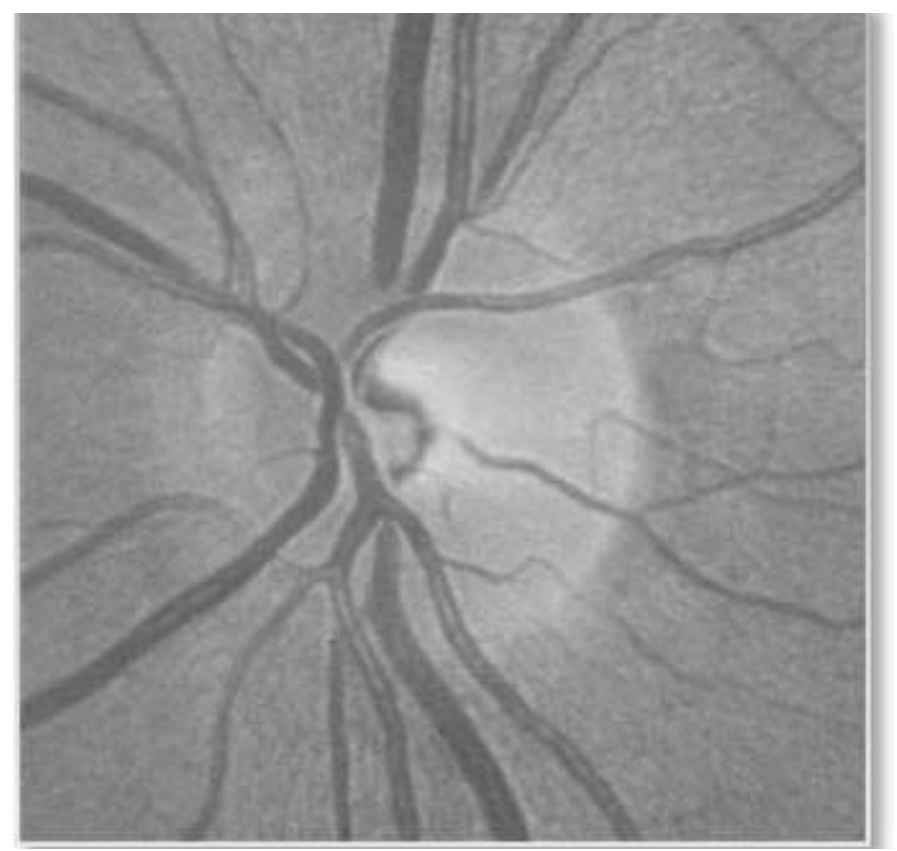

Fig. 2. The Optic Disc.

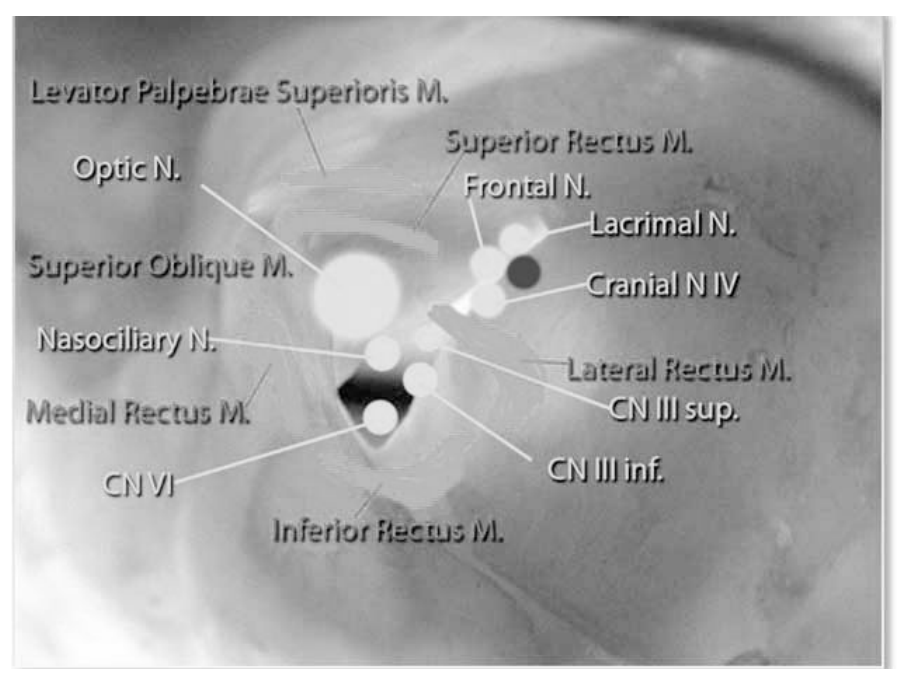

Fig. 3. Anatomy of the left orbital apex, demonstrating the extraocular muscle origins and the contents of the superior orbital fissure. Key: LPS,

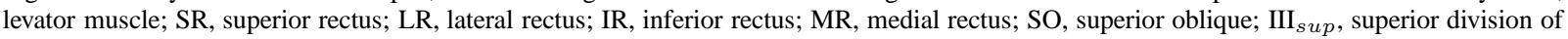
oculomotor nerve; $\mathrm{III}_{\text {inf }}$, inferior division of oculomotor nerve.

ing from lesions of the visual system posterior to the chiasm.

Behind the optic chiasm, the tracts pass posteriorly and most of the axons synapse in the layers of the lateral geniculate body (LGB) of the midbrain, with a minority passing to the superior colliculus and the Edinger-Westphal nuclei. The LGB is a posterolateral extension of the thalamus and contains synapses from retinal axons from both eyes, divided into magnocellular retinal ganglion cells and parvocellular retinal ganglion cells [6]. Of these, the fovea makes up approximately $50 \%$ of the surface area of the LGB, reflecting the importance of the fovea to vision in the human [7].

As described above, a small number of nerve fibers diverge to the Edinger-Westphal nuclei of the midbrain bilaterally to allow for parasympathetic innerva- 


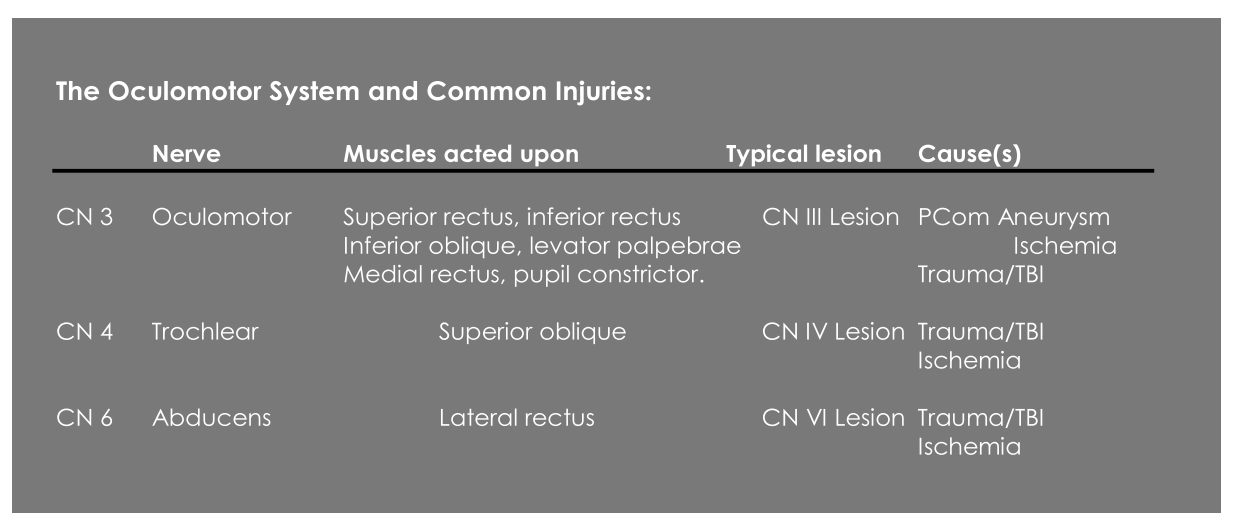

Fig. 4. The Oculomotor System and Common Injuries.

tion of the pupil (which subserves pupillary constriction), while the majority pass posteriorly to become the geniculo-calcarine tracts, which have both a parietal and temporal loop as they move back toward the occipital lobe.

The nerve fibers synapse again in the primary visual cortex, which has several names; the striate cortex, Brodeman's area 17, and visual area V1. The primary visual cortex in the occipital lobe is made up of columns corresponding to visual orientation, visual-spatial frequency and color information for small segments of the visual field. As a result, the primary visual cortex functions primarily as a topographic map of visual data rather than allowing the brain to interpret the information provided. Instead, conscious identification, visual memory and visual processing occur in the extrastriate cortex (visual association cortices).

It is the complexity of the system that allows for much of its robustness. Because visual field data is divided between both sides of the brain, damage to a portion of one occipital lobe does not completely blind the animal, but instead allows for continued function, albeit with some functional limitations because of the homonymous defect. Higher level visual discrimination is also possible at a very refined level, because the visual cortex allows for hierarchical distribution of function and integration with multiple levels of visual processing. This is based upon the complexity and integration of the Dorsal and Ventral visual pathways.

The Dorsal pathway is considered the "Where" pathway. It includes:

- Occipital lobe V3a for processing motion and direction

- Parietal lobes for processing depth and position to observer
- Posterior temporal / anterior occipital V5 for processing direction of movement

- Inferior temporal lobe and Fusiform gyrus for recognizing objects especially faces

- Frontal lobes for conscious perception

The Ventral visual pathway is considered the "What" Pathway

- Occipital V1 which receives signals from eyes

- Occipital V2 where complex shapes are registered

- Occipital V3 where angles and orientation are analyzed

- Occipital V4 which registers color and shape

\section{The oculomotor system}

Of equal importance to the human visual system as the ability to see is the ability to control the alignment of the eyes. This role is undertaken by a combination of neural subsystems, ranging from the frontal eye fields of the dorsal frontal lobes to the oculomotor, trochlear and abducens nuclei of the brainstem (Fig. 4).

Horizontal movement of the eyes begins in the bilateral frontal eye fields of the dorsal frontal lobes. This cortical region helps to initiate horizontal eye movements, and when impaired (such as in a patient with TBI or stroke) causes a tonic deviation of the eyes toward the side of the injury. Axons from this region project down to the abducens nucleus of the brainstem. Here, they synapse and then cross the midline as the medial longitudinal fasciculus (MLF) to synapse with the oculomotor nucleus. As a result, lesions of the MLF produce the so-called internuclear ophthalmoplegia (INO).

The oculomotor nucleus (Fig. 5) sends fibers anteriorly as the 3rd cranial nerve, which innervates the 


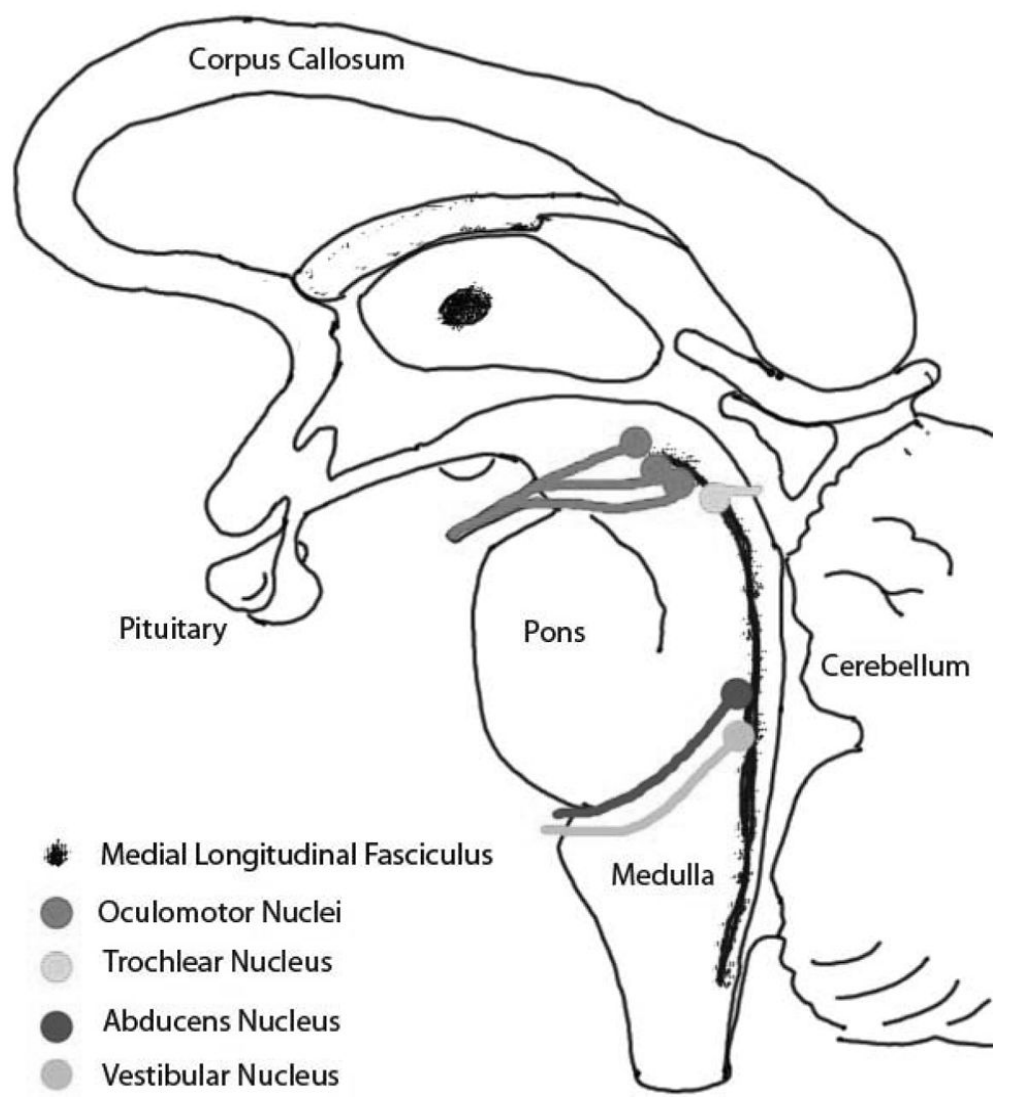

Fig. 5. The Brainstem Oculomotor Nuclei.

medial rectus, inferior rectus, superior rectus, levator palpebrae superioris, and inferior oblique muscles. An injury to this nerve produces a collection of problems, including ptosis and an eye held in abduction. Since the pupillary constrictor fibers are also carried on the 3rd nerve, lesions to this nerve can cause pupillary dilatation, which is an important pathognomonic finding in many compressive 3rd nerve lesions related to aneurysms of the posterior communicating artery. It is possible, however, to have pupil sparing 3rd nerve injuries.

The trochlear nucleus subserves the 4 th cranial nerve, which is involved in intorsion (also called incyclotorsion) when the eye is in the primary position, and inferior deviation of the eye when the eye is in adduction. This action is the result of the action of the only extraocular muscle that it innervates, the superior oblique. This muscle has the longest tendon of any of the extraocular muscles, running from the muscle belly, through the trochlea at the superior-nasal aspect of the orbital rim, then back to insert on the superior portion of the globe, but posterior to the equator. This insertion point leads to the unusual action of this muscle as a depressor of the globe despite its superior positioning (unlike the superior rectus, which is an elevator and excyclotorter of the globe).

The abducens nucleus acts through the 6th cranial nerve primarily to abduct the eye through the action of the lateral rectus muscle. The lateral rectus is a primary abductor of the eye, rotating the globe temporally. As described above, the lateral rectus is neurophysiologically linked to the medial rectus of the ipsilateral eye through the MLF, which allows the eyes to hold binocular fusion during horizontal pursuit. It is this ability to hold fusion which is taken for granted by humans, that is, until damage to the system in its multitude of forms "breaks" fusion and diplopia develops.

\section{The sympathetic visual system}

The sympathetic system provides much of the neural substrate for the so-called "fight or flight" mechanism which helps prepare us to meet the challenges of a 
potentially threatening situation. In the case of vision, the sympathetic system produces two distinct effects; dilation of the pupils (mydriasis) and elevation of the eyelid. Both of these functions appear to be directed at allowing more light into the eyes to help the organism to perceive a real or implied threat.

The sympathetic system describes a rather long and complex route through the human nervous system, and as a result is subject to trauma from multiple causes and at multiple levels. The sympathetic system originates in the hypothalamus, sending fibers down through the entire brainstem and into the cervical spinal cord to synapse at the Ciliospinal center of Budge in the upper thoracic spinal cord. Second-order neurons then pass out of the spinal cord and enter the thoracic ganglia of the sympathetic system, then run superiorly again until they reach the superior cervical ganglion (adjacent to the bifurcation of the common carotid artery), where they again synapse. As third-order neurons they form a fine latticework surrounding the internal carotid artery and enter the skull, sending out connections to the sudomotor (sweat) system of the face, transforming into the long ciliary nerves and eventually entering the eye to innervate the pupil dilator muscle. The fibers also innervate Muller's muscle (also called the superior tarsal muscle), a smooth muscle that helps the levator papebrae superioris to elevate the eyelid.

\section{The parasympathetic visual system}

By contrast to the sympathetic system of the eye, the parasympathetic system subserves the miotic reflex of the eye, allowing it to constrict the pupil to allow in less light and to protect itself when light intensity increases to an uncomfortable or dangerous level.

The parasympathetic system charts a much shorter and more direct pathway to the eye than the sympathetic system. It begins in the Edinger-Westphal nuclei of the midbrain, which join the oculomotor nerve (CN III) and pass anteriorly from between the thick columns of the descending cortico-spinal tracts (the cerebral peduncles). The nerves course anteriorly, through the cavernous sinus, and enter the orbit at the apex. The ocu- lomotor nerve then divests itself of the parasympathetic fibers, and these approximately 2,500 fibers synapse in the ciliary ganglion posterior to the globe. The ciliary ganglion sends post-ganglionic fibers forward to the eye as the short ciliary nerves, which synapse in the eye with the pupillary constrictor muscle and subserve the miotic reflex.

\section{Summary}

Although Thoreau admonishes us that it is not what you look at that is important, I hope it is clear from the discussion above that what you look at directly affects what you see, making the two aspects of vision inseparable.

As a result of this intertwined nature, healthy functioning of all of the system's representative parts is necessary for the visual system to work properly. But vision does not reside only in the eyes. I hope it is clear from the previous discussion that the human visual system is a very complicated network of afferent and efferent connections spread through several regions of the head, neck and upper thorax.

It also follows that, because of the complexity of the visual system, it is very subject to trauma. As a result, it is very common for victims of motor vehicle accidents, accidental trauma, sports-related injuries, and domestic altercations to present with a visual complaint.

\section{References}

[1] R. Dawkins, The Greatest Show on Earth: The Evidence for Evolution, (1st ed.), Bantam Books, 2009.

[2] R. Snell, Clinical Anatomy of the Eye, (2nd ed.), M.A. Malden, ed., Wiley-Blackwell, 1997.

[3] J. Chan, Optic Nerve Disorders, (1st ed.), New York: Springer, pp. 130-131.

[4] Ibid.

[5] J. Chan, Optic Nerve Disorders, (1st ed.), New York: Springer, p. 132.

[6] J. Trobe, The Neurology of Vision, (1st ed.), Oxford: Oxford University Press, pp. 32-33.

[7] Ibid. 